\title{
Metodología para el estudio del brand-placement con enfoque de género: más allá del análisis de contenido
}

Miriam Redondo-Rodríguez y Natalia Papí-Gálvez | Natalia.p@ua.es

UniVERSIDAD DE ALICANTE

Resumen: La mayoría de los estudios realizados sobre el tratamiento de género en la publicidad audiovisual se ciñen al formato convencional. Los nuevos formatos no se adecuan siempre a estos patrones. Con este fin, se ha elaborado un protocolo de análisis para estudiar la estructura del brandplacement, como forma no convencional de publicidad, en temas de género en la ficción española. Esta herramienta ha sido sometida a rigurosos controles de fiabilidad y validez con el ánimo de garantizar la confiabilidad y rigurosidad científica de las mismas, tanto en su composición como en su aplicación.

Palabras clave: Fiabilidad, Brand-placement, estereotipos de género, análisis de contenido

Abstract: Most studies on the treatment of gender in the audiovisual advertising follows the conventional format. The new formats do not conform to these standards. With this aim in mind an analytical protocol has been developed to study the structure of the brand-placement, as a non-conventional advertising, concerning gender issues in the Spanish fiction. This tool has been subjected to rigorous checks of reliability and validity with the aim of ensuring their trustworthiness and scientific accuracy, both in its composition and in its implementation.

Key words: Reliability, Brand-placement, gender stereotype, content analysis 


\section{Introducción}

El presente artículo procede de un proyecto que pretende discernir los límites de actuación y principios seguidos por la técnica publicitaria del brand-placement en materia de género, y en el ámbito de la ficción española. Para ello es pertinente el diseño y la validación de determinadas herramientas que se adapten a las características de la técnica y éste es el fin que se persigue en esta ocasión.

Desde que en la década de los setenta McArthur y Resko (1975) formularan una categorización de estereotipos en la publicidad convencional, la proliferación de estudios e investigaciones sobre esta temática ha sido notable. En España, este tipo de estudios sobre la imagen de las mujeres comienza a realizarse a finales de la década de los 80 (Ceulemans y Fauconnier, 1980; Galán, 2007; Hernández, 2006) debido, en parte, a que los medios de comunicación, y especialmente el medio televisivo, tienen un protagonismo especial en la construcción social de la realidad, constituyendo uno de los agentes fundamentales de socialización de nuestra época junto con la familia y la escuela (Santiso, 2001; Meriño, 1998). La continua exposición a ellos hace que recalen hasta el más recóndito espacio de nuestra sociedad, siendo uno de los agentes contribuyentes a la configuración de la cultura de masas, que dota al grupo de sus señas de identidad (Vilches, Berciano y Lacalle, 1999).

La capacidad de congregar elevadas audiencias de la televisión y su carácter audiovisual le convierte en un medio sobresalientemente atractivo para la inserción de acciones publicitarias que, en busca de la eficacia comercial, han pasado de tener un carácter intersticial a ubicuo, invadiendo el resto de contenidos y creando formas publicitarias que escapan a las definiciones y legislación del formato convencional (Méndiz, 2000; Reinares, 2007). Además, el nuevo sistema de consumo y la crisis del modelo convencional publicitario (Reinares, 2007; Fernández, 2005; Farré, 2005) han acelerado un cambio de la publicidad que comienza a hacer mella en España en la década de los 90.

De este modo, surgen técnicas no convencionales que escapan a los cánones establecidos históricamente para las comunicaciones comerciales. Algunas de estas formas plantean una ventaja legal ya que, al presentarse en plena fusión con los contenidos programáticos, todavía no computan en los límites de tiempo establecidos para la publicidad y aumentan, así, la rentabilización de la parrilla (Pérez, 2005).

A este respecto, uno de los formatos más ampliamente utilizados por la industria es el brand-placement. La utilización de esta técnica ha sido creciente desde que se origina en la década de los 40 en Estados Unidos y en la década de los 90 en España. El brand-placement es ampliamente utilizado por los anunciantes, en buena parte, por las ventajas que presenta en términos de comunicación (Méndiz, 2000). A través de la misma, la marca se asocia a un entorno familiar. Existe una carencia de ruido en el entorno en que se desarrolla la acción publicitaria que beneficia la atención y la comprensión. El espectador se muestra predispuesto a ver el programa y puede aceptar mejor el mensaje que en el caso de la 
exposición al corte publicitario tradicional. También constituye un reflejo de situaciones de compra y consumo, de modo que se puede realizar una publicidad más eficaz y que informe y propicie la identificación a la vez. Es más, los datos para el periodo 2005-2010 (PQ Media, 2006) prevén un crecimiento del $27,9 \%$ en el gasto en brand-placement, lo que supondría alcanzar los 7.550 millones de dólares. A su vez, el crecimiento de esta modalidad publicitaria continúa superando de forma significativa el del marketing y la publicidad tradicionales. La televisión es el medio que mayor incremento ha experimentado en la inversión referente a esta técnica.

El brand-placement ha sido especialmente utilizado en las series de ficción (i.e. Del Pino y Olivares, 2006). Este producto audiovisual también ha visto crecer su demanda en España en los últimos años (Vilches, Berciano y Lacalle, 1999; Vilches, 2007). Las series de ficción es uno de los géneros preferidos tanto por mujeres como por hombres de cualquier edad (Instituto de la Mujer, 2007) por lo que a las ventajas anteriormente nombradas del brandplacement, se les debe añadir la doble capacidad socializadora que la serie de ficción confiere a la técnica (Castelló, 2006).

Por un lado, las teleseries, si bien constituyen un producto óptimo para promover el cambio social, dado que son transmisoras de patrones de conducta, modas y estructuras sociales, también reproducen estereotipos. Por otro lado, es una característica de la publicidad utilizar imágenes de rápida identificación y, por tanto, la estereotipación de rasgos (Instituto de la Mujer, 2007; Galán, 2007; Charles, 2002). Así, la publicidad en las series de ficción son un buen escaparate para el tratamiento de temas relacionados con la mujer (Galán, 2007).

En este sentido, si bien son numerosos los estudios que ponen de manifiesto la presencia de estereotipos de género en la publicidad convencional (Peña-Marin y Fabretti, 1994; de Pablos, 2004; Charles, 2002; Furnham y Mak, 1999; García, 2003; Navarro, 1984; Sánchez, García, Grañido y Berganza, 2002) es pertinente y necesario realizar nuevas herramientas de recogida de información que permitan indagar en este tipo de datos adaptándose a las características de los nuevos formatos y técnicas publicitarias.

El carácter relativamente novedoso del brand-placement provoca que se trate de un tema sobre el que se han realizado pocos estudios con enfoque de género en nuestro país. Por las propias características de este formato, las herramientas de medición utilizadas deben relacionar diversos campos (publicidad, televisión y ficción) y a su vez, mediante su combinación, suponer instrumentos útiles y válidos para el análisis del brand-placement.

\section{Estrategia metodológica e instrumentos de recogida de información}

El objetivo específico de este estudio consiste en diseñar una metodología y unas herramientas adecuadas, encaminadas a analizar el tratamiento de género en las series de ficción españolas por medio de la observación de la estructura del brand-placement. Para ello, se aplica de forma reiterativa los controles diseñados para conseguir instrumentos de 
recogida de información válidos, incluida una primera aplicación que suponga un pre-test para futuras aplicaciones.

El análisis de contenido constituye el método central de la investigación. Desde sus orígenes, este método ha procurado establecer criterios adecuados de validez, lo que le ha conferido madurez como técnica (Krippendorff, 1990). En la actualidad, es uno de los más utilizados en las investigaciones llevadas a cabo en el ámbito de la comunicación mediática y es el más extensamente empleado para el estudio de la imagen de las mujeres en los medios de comunicación (Ceulemans y Fauconnier, 1980; Méndiz, 2005; Galán, 2007; Instituto de la Mujer, 2007; De Pablos, 2004; Berganza, 2006, Espinar, 2006; Del Moral, 2000). Este método analiza la comunicación de forma sistemática, objetiva y cuantitativa, con la finalidad de medir determinadas variables (Kerlinger, 1986). En este estudio se utiliza con el objeto fundamental de evaluar y calibrar la imagen de un grupo social, las mujeres, en un medio masivo, la televisión, y en una técnica publicitaria no convencional, el brand-placement.

Ahora bien, no es posible obviar que la marca anunciada hace uso de los personajes de la serie, de los escenarios en los que aparece y de las situaciones que se producen. Tales elementos ayudan a contextualizar $y$, por tanto, a interpretar adecuadamente las identidades, roles y relaciones de género que se refuerzan o transmiten a través de la publicidad. El análisis debe extenderse más allá de la escena en la que aparece la marca. Debe profundizar en otros elementos propios de la serie de ficción relevantes desde un enfoque de género. La triangulación de técnicas facilita una mayor comprensión del objeto de estudio (i.e. Castelló, 2006; Instituto de la Mujer, 2007). En este caso se propone un análisis documental y un análisis narrativo.

La información que proporciona el análisis documental atiende a la identificación de la serie estudiada como contexto en el que tienen lugar las acciones publicitarias. Permite, asimismo, el recuento y posición de los personajes sobre los que se aplicará el análisis narrativo. El narrativo permite observar si se emplea algún estereotipo pese a que en el momento del emplazamiento no se haga de forma explícita, al esclarecer y describir el perfil de los personajes en la serie de ficción. La información que debe proporcionar el análisis narrativo de los personajes es imprescindible para averiguar la relación entre el emplazamiento publicitario y el personaje y si esto obedece o no a cuestiones de género.

Forma parte del análisis documental tres tipos de preguntas (tabla 1). Un primer grupo estaría formado por aquellas variables relacionadas con la definición del universo y con la selección de la muestra (P1, P2, P5-P10, P18). El segundo grupo queda constituido por aquellas variables y apartados que proporcionan información básica sobre el contenido de la serie de ficción, que actúa como soporte de la publicidad no convencional analizada (P3, P4, $\mathrm{P} 14, \mathrm{P} 15)$. Un tercer y último grupo estaría formado por aquellas variables que proporcionan información relevante desde una perspectiva de género sobre el contexto en el que se desarrolla la publicidad no convencional (P11-P13, P16, P17). En concreto, la P11 y la P12 permiten conocer el número de emplazamientos realizados por cada sexo y saber si son 
representativos de la realidad mediante un análisis de bondad de ajuste. Esta métrica se ha llevado a cabo anteriormente para contabilizar la presencia de hombres y mujeres en publicidad convencional en muchos estudios (Charles, 2002; Peña-Marín y Fabretti, 1994). Asimismo, la P13 contribuye a discernir la posición o el papel que desempeña la mujer en la ficción española. Ha sido aplicada en los estudios sobre publicidad convencional (Furnham y Mak, 1999; De Pablos, 2004) y en algunos que analizan el contenido de las teleseries (Galán, 2007; Instituto de la Mujer, 2007).

Con el análisis narrativo (tabla 2) se visualiza el rol de cada personaje y el tipo de tramas en las que están involucrados (Instituto de la Mujer, 2007; Charles, 2002; Galán, 2007). También ayuda a la identificación de los estereotipos de género a través del aspecto físico de los mismos, y de sus rasgos psico-sociales (De Pablos, 2004; Berganza, 2006; Instituto de la Mujer, 2007; Galán, 2007). Para finalizar, se introducen tres variables destinadas a calibrar el peso que los estereotipos de género tradicionales tienen en el elenco de las series estudiadas (P15, P16 y P17). También en esta ocasión, la propuesta que se trae es el resultado de la combinación de las categorizaciones presentadas por estudios tanto de series de ficción como de publicidad convencional (Suárez, 2006; De Pablos, 2004; Berganza, 2002, Furnham y Mak, 1999; del Moral, 2000; Altés, 2001; Charles, 2002; García, 2003; Sánchez, García, Grandío y Berganza, 2002; Shibley, 1995; Vigil, 1994; Peña-Marín y Fabretti, 1994; Quispe, 2006; Rey, 1999).

Una vez analizadas las producciones seleccionadas y los personajes que de ellas toman parte se realiza el análisis de contenido de las acciones de brand-placement (tabla 3). Se proponen dos variables de ubicación espacial (P5 y P6) ambas utilizadas en la mayoría de estudios revisados. Otro grupo se centra en el personaje que realiza la acción, para averiguar si realiza una tarea tradicionalmente atribuida a su sexo (Peña-Marín y Fabretti, 1994; Charles, 2002; Méndiz, 2001; Instituto de la Mujer, 2007). Se indica el rol empleado en la acción (Furnham y Mak, 1999) y el tipo de emplazamiento que realiza (Del Pino y Olivares, 2006) para tratar de calibrar la relevancia y el nivel de autoridad conferido. Se incluye una variable sobre prescripción directa a la compra por parte del personaje, por si hay diferencias de género según el mensaje. El número de personajes que intervienen en el emplazamiento actúa como otro indicador de autoridad y poder de convocatoria del personaje central. Por último, se incluyen dos variables para conocer el tipo de producto y la marca, con el ánimo de discernir si existe un empleo diferenciado de la técnica por sexos.

\section{Control de calidad}

Los controles de calidad persiguen que el estudio pueda ser aceptado por la comunidad científica por cumplir con una serie de condiciones. Estas condiciones se resumen en que los resultados sean fieles aproximaciones a la realidad estudiada (Ruiz Olabuénaga, 1998:115). Tanto fiabilidad como validez son cruciales en el análisis de contenido (Krippendorff, 1990:191). En estos controles, y por la peculiaridad del objeto de estudio de las ciencias sociales, entra en juego el juicio del investigador y de los codificadores (Gómez, 2000). 
En este estudio, los controles no se circunscriben a pruebas aplicadas exclusivamente a los instrumentos de recogida de información. Los controles se establecen en todas las etapas del mismo (pre-estudio, estudio y post-estudio) (Ruiz Olabuénaga, 1998: 118-120) lo que redunda, sin duda, en la calidad de la propuesta (tabla 4).

Como parte del pre-estudio, se advierte que los instrumentos de recogida de información pertenecen a una investigación original centrada en la ficción española. Este ámbito geográfico responde al propio objeto de estudio, ya que cada país tiene su propia cultura de masas. De esta forma, tanto guionistas como público comparten esta realidad social. En esta línea, se establecen una serie de criterios que definen, en última instancia, el universo, tales como: la temporalidad (se acota el periodo de estudio a la emisión anual); la actualidad (sus tramas han de ser coetáneas a la realidad para no sesgar los estereotipos por las series que están ambientadas en otra época); el alcance (las producciones deben situarse en su segunda temporada lo que garantiza la aceptación de las mismas por parte de la audiencia y deben haber alcanzado un $20 \%$ de cuota de pantalla); la representatividad (deben ser emitidas en cadenas de emisión en abierto y de ámbito nacional) y la homogeneidad (todas deben emitirse en prime-time con periodicidad semanal). Además, se aplica un muestreo de intervalo consistente en analizar los cinco últimos capítulos de las producciones seleccionadas por cumplir con los anteriores criterios (cinco series de ficción) para, así, obtener una visión prolongada que permita observar el desarrollo de la técnica y no limitarla a acciones puntuales. En el análisis concreto de la publicidad, se propone eliminar del estudio las repeticiones, es decir, los emplazamientos que son realizados por el mismo personaje y de la misma marca, que corresponden a una misma acción publicitaria para no sobreestimar la presencia de ciertos contenidos.

Los anteriores criterios de definición del universo y los procedimientos seguidos para la selección de la muestra obedecen en todo momento al marco teórico, a las hipótesis, a los objetivos de la investigación y a un razonamiento lógico y adaptado a las características del formato estudiado. Esto contribuye a dotar al estudio de validez externa potenciando su extrapolación y representatividad. Igualmente, muchas de las variables que forman parte de los instrumentos son tomadas de estudios sobre publicidad convencional cuya confiabilidad ha sido probada con anterioridad, lo que incrementa per se el grado de validez externa, ya que garantiza su extrapolación. Otras preguntas son adaptaciones de este tipo de variables o nuevas, creadas a partir de la literatura revisada relacionada con el brand-placement, cuya validez y fiabilidad necesitan ser medidas.

En la etapa de estudio, se realizan ensayos previos y pruebas de fiabilidad (test-test) sobre un porcentaje del material de la muestra (10\% de los capítulos y el $20 \%$ de los personajes) en aquellos instrumentos que contienen preguntas cuya contestación pudiera estar sujeta a la apreciación del codificador (análisis narrativo y de contenido). Se debe añadir que las sesiones han sido realizadas por dos codificadores, en intervalos de dos horas y de forma aislada para evitar los elevados índices por acuerdo y disminuir la coincidencia por fatiga. 
Ciertamente, para medir la reproductibilidad y estabilidad de los instrumentos existen numerosas formas de calcular la fiabilidad intercodificadores, cuya aplicación puede depender del tamaño de la muestra, de la cantidad de codificadores y del tipo de variables. En este caso, se opta por la aplicación del índice "alfa" de Krippendorf (1990) en variables de intervalo (discretas o continuas). Para las variables nominales y ordinales (que son prácticamente la totalidad en este estudio) se calculó el índice "Pi" de Scoot (Krippendorf, 1990) apto para los supuestos en los que participan dos codificadores. Estos estadísticos no se ven afectados por el tamaño de la muestra para la determinación del grado de confiabilidad. Por convención se estima que índices superiores a un $75 \%$ para el empleo de estas dos fórmulas son adecuados (Wimmer y Dominich, 1996). Los datos de estos estadísticos son contrastados con la observación del simple porcentaje de acuerdo entre jueces y los coeficientes de correlación " $\rho$ de Spearman" y "V de Kramer" que miden el grado de asociación entre dos variables a nivel ordinal y nominal respectivamente (tabla 5).

Finalmente, en la etapa de post-estudio, se ha medido otro tipo de fiabilidad conocida como estabilidad. Transcurridos cuatro meses desde la primera codificación, con motivo de evitar el recuerdo espontáneo, uno de los codificadores que participa en el proceso vuelve a aplicar la misma herramienta a la misma muestra (test-reset). Así, se averigua el grado de permanencia, es decir, de estabilidad en la catalogación de un mismo hecho a lo largo del tiempo con base a un mismo criterio. Se utilizan en este caso los mismos índices que se emplearon para medir la fiabilidad intercodificadores en los mismos términos en los que fueron aplicados con anterioridad. Una vez calculados todos los estadísticos, estos muestran asociaciones fuertes en todos los casos (tabla 5).

\section{Conclusiones}

Con todo lo expuesto se realiza un aporte metodológico a futuras investigaciones relacionadas con la técnica del brand-placement. El empleo de diversas técnicas de análisis aplicadas a un mismo objeto de estudio posibilita una mayor y más precisa obtención de datos así como su contraste, facilitando, además, el establecimiento de relaciones y análisis de los efectos de los medios en la sociedad.

En este caso, el hecho de emplear los tres análisis (documental, narrativo y contenido) permite que cada uno de ellos profundice en uno de los pilares básicos del brand-placement, ya sea producto (contenido), personaje (narrativo) o soporte (documental), con una mayor funcionalidad dado que no es posible, en la técnica estudiada, la disociación de los contenidos programáticos y publicitarios. Se pretende aumentar, así, la eficacia del método, obteniendo mayor volumen de información, más exacta y con menor esfuerzo de codificación, por lo que contribuye a la precisión del estudio y a la parsimonia.

Cabe señalar que los conceptos utilizados en la elaboración de los instrumentos se desprenden de una base teórica previa y que, por tanto, se adecuan a lo establecido en el campo estudiado. En todo momento se han introducido conceptos referidos a la técnica del 
brand-placement, siempre atendiendo a clasificaciones globales, aceptadas y establecidas con anterioridad. La introducción de otro tipo de conceptos supondría una pérdida de avales y la necesidad de redefinir términos que ya existen, perdiendo la gran ventaja que ofrece la acumulación del conocimiento científico. En esta ocasión, además, se trata de un estudio interdisciplinar (audiovisual, publicidad y estudios de género) y que, por tanto, le precede una importante base teórica. Tras los controles de calidad y habiendo superado las pruebas de confiabilidad establecidas con índices satisfactorios se concluye en considerar los instrumentos testados válidos y por tanto extrapolables y generalizables a estudios con propósitos similares.

Por último, cabe poner de manifiesto la importancia de realizar este tipo de estudios metodológicos en los que no solamente se marca la estrategia, sino que se construyen los instrumentos y se testa su fiabilidad y validez, antes de ser aplicados a muestras superiores. Con estos estudios se hace posible la detección de errores, tanto de método como de aplicación, posibilitando su modificación y perfeccionamiento. Por tanto, se trata de obtener un diseño metodológico adecuado, así como unos instrumentos fiables y válidos para los objetivos marcados, de modo que sea posible su aplicación por otras investigaciones con una calidad científica garantizada. 


\section{REFERENCI AS BI BLI OGRÁFI CAS}

ABERCROMBIE, Nicholas (1998): Diccionario de sociología, Madrid, Cátedra.

ALTÉS, Elvira (2004): "La publicidad ante el nuevo imaginario femenino: impulso o retroceso", Sevilla, Instituto Andaluz de la Mujer, Observatorio andaluz de la publicidad no sexista.

AIMC (2007): Resumen general EGM, Madrid, AIMC, en: http://www.aimc.es.

ANDREU, Jaime (2001): "Las técnicas de análisis de contenido: Una revisión actualizada", www.centrodeestudiosandaluces.es (consultado el 15/10/2009)

BABBIE, Earl (2000): Fundamentos de la investigación social, Madrid, International Thomson editores.

BERGANZA CONDE, Ma Rosa y RUIZ SAN ROMÁN, Jose A. (2005): Investigar en comunicación, Madrid, Mc Graw Hill.

BERGANZA, Ma Rosa; GARCÍA, Carmela y GANDÍO, Ma Mar (2002): "Women and advertising: evolution of stereotypes in Spanish mass media", 23 Conferencia y Asamblea General AlECS, Barcelona, Institut de la comunicació de la Universidad Autónoma de Barcelona, Comunicación intercultural.

BRUNET, Ignasi; PASTOR, Inma y BELZUNEGUI, Angel (2002): Tècniques d'investigació social. Fonaments epistemològics I metodològics, Barcelona, Universitat Rovira y Virgili, Pòrtic.

CASTELLÓ, Enric (2006): "La construcción nacional en las series de ficción: visión sobre una década de producción de Televisió de Catalunya", Quaderns del CAC, no 23-24 pp. 205-216.

CASTELLÓ, Enric (2004): "Mecanismos de construcción de la identidad cultural en las series de ficción: el caso de la televisión autonómica en España", Revista de estudios sobre culturas contemporáneas, vol.10, n²0, pp 46-73.

CASTELLÓ, Enric (2006): "El análisis de las series televisivas como creadoras de modelos sociales y discursos ideológicos: una evaluación metodológica", IX Congreso Ibercom, Asociación Iberoamericana de comunicación y Universidad de Sevilla.

CHARLES, Mercedes (2002): "La televisión y la construcción de género", II Congreso de Imagen Pedagógica, Mazatlán, Sinaloa.

CIS (1984): “Mujer y Publicidad". Estudio no 1.384, enero, Madrid, CIS. 
DE PABLOS, Jose M. (dir.) (2004): "Los nuevos modelos de mujer y de hombre de la publicidad televisiva actual", Revista Latina de Comunicación Social, Universidad Carlos III y Universidad Complutense de Madrid. http://www.cac.cat/pfw_files/cma/recerca/estudis_recerca/modelosmujerho mbre.pdf (consultado el 04/03/2010)

DEL MORAL, Esther (2000): "Los nuevos modelos de mujer y de hombre a través de la publicidad", Comunicar, marzo no 14, pp. 208-217.

DEL PINO, Cristina (2006): "El brand-placement en seis series españolas de Farmacia de Guardia a Periodistas: un estudio empírico", Revista Latina de Comunicación Social, no 61, II época, de enero-diciembre, en http://www.ull.es/publicaciones/latina/200617delPino.pdf (consultado el 20/02/2010).

DEL PINO, Cristina y OLIVARES Fernando (2006): Brand Placement: integración de marcas e la ficción audiovisual. Evolución, casos, estrategia y tendencias, Madrid, Gedisa editorial.

DEL RINCÓN, Deilo; ARNAL, Justo; LATORRE, Antonio y SANS, Antoni (1995): Técnicas de investigación en ciencias sociales, Madrid, Dykinson.

ESPINAR RUIZ, Eva (2006): "Análisis y estereotipos de género en la programación y en la publicidad infantil. Análisis cuantitativo", Revista Latina de Comunicación Social, no 61, II época, de enero-diciembre, en: http://www.ull.es/publicaciones/latina/200614EspinarRuiz.pdf (consultado el $5 / 11 / 2009)$

FARRÉ COMA, Jordi (2005): "Catalogación provisional de los formatos publicitarios no convencionales", Quaderns del CAS, n० 22, pp. 21-34.

FERNÁNDEZ CAVIA, Josep (2005): "Los formatos no convencionales y la transformación del negocio publicitario en la televisión", Quaderns del CAS, no 22, pp. 35-48.

FURNHAM, Adrian y MAK, Twiggy (1999): "Sex-role Stereotyping in Television Commercials: A review and comparison of Fourteen Studies Done on Five Continents Over 25 Years - Statistical data Included", Sex roles: Journal of Research, September en http://findarticles.com/p/articles/mi_m2294/is_ 1999_Sept/ai_58469478/ (consultado 04/01/2010)

FURNHAM, Adrian y SAAR, Aaar (2005): "Gender-role Stereotyping in adult and children's television advertisements: a two-study comparison between great a Britain and Poland", Communications, Vol. 30, no 1, pp 73-90. 
FURNHAM, Adrian; BABITZKOW, Matte; y UGUCCIONI, Smerelda (2000): "Gender stereotyping in television advertisements: a study of French and Danish television", Genetic, Social, and General Psychology Monographs, no 126, pp. 79-104.

FURNHAM, Adrian y VOLI, Virginia (1989): "Gender stereotypes in Italian television advertisements", J ournal of Broadcasting and Electronic, Media, no 989, pp. 175-185.

GALÁN, Elena (2007): La imagen social de la mujer en las series de ficción, Cáceres, Universidad de Extremadura.

GARCÍA FERRANDO, Manuel (1992): Socioestadística. Introducción a la estadística en sociología, Madrid, Alianza Universidad Textos.

GÓMEZ MENDOZA, Miguel A. (2000): "Análisis de contenido cualitativo y cuantitativo. Definición, clasificación y metodología", Revista de ciencias humanas, no 20, pp 12.

GONZÁLEZ DEL RÍO, Ma José (1997): Metodología de la investigación social. Técnicas de recolección de datos, Alicante, Amalgama.

HERNÁNDEZ, Yolanda (2006): "Acerca del género como categoría analítica", Nómadas. Revista crítica de ciencias sociales y jurídicas, no 13.

INSTITUTO DE LA MUJER (1990): La mujer en la publicidad, Madrid, MTAS.

INSTITUTO DE LA MUJER (2003-2006): Informes 2003-2006, Observatorio de la publicidad sexista, Madrid, MTAS.

INSTITUTO DE LA MUJER (2007): Tratamiento y representación de las Mujeres en las teleseries emitidas por las cadenas de televisión de ámbito nacional, Madrid, MTAS.

INSTITUTO OFICIAL DE RADIO Y TELEVISIÓN (RTVE) (2002): Mujer, violencia y medios de comunicación, Dossier de prensa, Madrid, MTAS.

KERLINGER, Fred. N. (1986): Fundations of behavioral research (3a Edición), Nueva York, Holt, Rinehart y Winston, p. 21.

KRIPPENDORFF, Klaus (1990): Metodología de análisis de contenido: teoría y práctica, Barcelona, Paidós comunicación.

KWANGOK, Kim (2005): "Television commercials as a lagging social indicator: gender role stereotypes in Korean television advertising". Sex Roles: A J ournal of Research. December.

LANDRY, Réjean (1998): "L'analyse de contenu", Recherche sociale. De la problemátique à la collecte des données. Benoit Gauthier (Editor). Sillery, Presses de I'Université du Québec. pp. 329-356.

MCARTHUR, L. Z.; RESKO, B. G. (1975): "The portrayal of men and women in American TV commercials", J ournal of Social Psychology, 97, pp. 209-220. 
MÉNDIZ NOGUERO, Alfonso (2000): Nuevas formas publicitarias. Patrocinio, product placement, publicidad en Internet. Málaga, Universidad de Málagamanuales.

MÉNDIZ NOGUERO, Alfonso (2005): Publicidad, educación y nuevas tecnologías. Informe elaborado para el centro nacional de información y comunicación educativa (C.N.I.C.E), Madrid, Ministerio de Educación y Ciencia.

MERIÑO, Adriana (1998): "El rol de la interacción ficcional televisiva en la formación de identidades", IV Congreso Latinoamericano de Ciencia de la Comunicación, Brasil, RECIFE-Brasil.

NAVARRO, Beatriz et. al (1984): La imagen de loa mujer en la televisión española 1984, un guión entre lo real y lo imaginario, Madrid, Instituto de la Mujer.

PEÑA-MARÍN, Cristina y FABRETTI, Carlo (1994): La mujer en la publicidad, Madrid, Instituto de la Mujer, MTAS.

PÉREZ RUIZ, Miguel A. (2005): "Nuevas formas de publicidad en la Televisión sin Fronteras. Una reconsideración como técnicas de comunicación publicitaria", Telos, no 65, segunda época, Octubre-Diciembre.

PQMEDIA (2006): Country By Country Product Placement Forecast Through 2010, www.pqmedia.com (consultado 02/02/2010)

QUISPE LÁZARO, Arturo (2006): “Estereotipos masculinos en la publicidad”, en http://www.europrofem.org/contri/2_05_es/es-masc/66es_mas.htm (consultado 02/02/2010).

QUIVY, Raymond y CAMPENHOUDT, Luc V. (1997): Manual de recerca en Ciències Socials, Barcelona, Herder.

REINARES LARA, Pedro J.; REINARES LARA, Ricardo y REINARES LARA, Eva Marina (2007): "Valoración de la eficacia en las formas publicitarias no convencionales en televisión: un estudio exploratorio", en AYALA CALVO, Juan C. (coord.): Conocimiento, innovación y emprendedores: camino al futuro, La Rioja, Universidad de La Rioja, pp 14.

REY, Juan (1999): "El nuevo arquetipo (masculino) para un nuevo consumidor (masculino)", Comunicar, marzo, n०1, pp 61-69.

RITZER, George (1996): Teoría sociológica contemporánea, Madrid, Mc Graw Hill.

RUIZ OLABUÉNAGA, Jose I.; ARISTEGUI, Iratxe y MELGOSA, Leire (1998): Cómo elaborar un proyecto de investigación social. Cuadernos monográficos del ICE, Bilbao, Universidad de Deusto.

SÁNCHEZ ARANDA, José J; GARCÍA ORTEGA, Carmela; GRANDÍO PÉREZ, Ma Mar y BERGANZA CONDE, Ma Rosa (2002): El espejo mágico. La nueva imagen de la mujer en la publicidad. Instituto Navarro de la Mujer, Pamplona, Instituto Navarro de la Mujer, Gobierno de Navarra. 
SANTISO SANZ, Raquel (2001): "La mujer en la publicidad: análisis, legislación y aportaciones para un cambio", Acciones e investigaciones sociales, no 13, pp. 43-60.

SHIBLEY HYDE, Janet (1995): Psicología de la mujer: la otra mitad de la experiencia humana, Madrid, Morata.

SUÁREZ VILLEGAS, Juan C. (2006): La Mujer Construida: Comunicación e Identidad Femenina, Madrid, Ed. Mad.

TORRANO PALAZÓN, Jose y FLORES LÓPEZ, Enrique (2005): "Principales factores determinantes de la actitud hacia el emplazamiento del producto", VIII Forum Internacional sobre Las Ciencias, las Técnicas y el Arte Aplicadas al Marketing. Academia y Profesión, Universidad Complutense, Madrid, pp 605 $-627$.

CEULEMANS, Mike y FAUCONNIER, Gido (1980): Imagen, papel y condición de la mujer en los medios de comunicación social. Compilación y análisis de los documentos de investigación, París, Organización de Naciones Unidas. UNESCO.

VIGIL, Mariló (1994): "Armas de mujer, armas de hombre", en PEÑA-MARÍN, C. y, FABRETTI, C. La mujer en la publicidad, Instituto de la Mujer, Madrid, pp $132-135$.

VILCHES, Lorenzo; BERCIANO, Rosa y LACALLE, Charo (1999): "La ficción nacional, por fin a escena", Anàlisi, no 23, pp 25-57.

VILCHES, Lorenzo (2007): Culturas y mercados de la ficción televisiva en I beroamérica, Anuario Obitel, Barcelona, Gedisa, pp 279.

WIMMER, Roger D. y DOMINICK Joseph. R. (1996): La investigación científica de los medios de comunicación. Una introducción a sus métodos, Barcelona, Bosch Comunicación. 


\begin{tabular}{|c|c|c|}
\hline \multicolumn{2}{|c|}{ Preguntas y variables } & \multirow{2}{*}{$\begin{array}{l}\text { Categorías } \\
\text { (Serie) }\end{array}$} \\
\hline P1 & Nombre & \\
\hline P2 & Productora & (Nombre productora) \\
\hline P3 & Temática & Laboral, Familiar, Juvenil, \\
\hline P4 & Género & Drama, Comedia, Dramedia, Trhiller, Policiaco, Otro \\
\hline P5 & $1^{\circ}$ emisión & DD/MM/AAAA \\
\hline P6 & Temporada actual & (No de temporada) \\
\hline P7 & Duración media por capítulo & (En minutos) \\
\hline P8 & Cadena emisión & (Nombre) \\
\hline P9 & Audiencia media & \multirow{2}{*}{ (Datos fuente Sofres) } \\
\hline P10 & Share medio & \\
\hline P11 & No de personajes femeninos & (Número) \\
\hline P12 & No de personajes masculinos & (Número) \\
\hline P13 & Sexo del protagonista & Hombre, Mujer \\
\hline P14 & Ficha técnica & Creación, Dirección, Guión \\
\hline P15 & Sinopsis & (Codificación abierta) \\
\hline P16 & Organigrama & $\begin{array}{l}\text { (Codificación abierta. Recoge información de las relaciones } \\
\text { jerárquicas entre personajes) }\end{array}$ \\
\hline P17 & Escenarios principales & (Codificación abierta) \\
\hline P18 & Total de emplazamientos & $\begin{array}{l}\text { (No emplazamientos figura central, con personaje) } \\
\text { (No emplazamientos pasivos secundarios sin figura central) }\end{array}$ \\
\hline
\end{tabular}

Tabla 1. Preguntas-categorías análisis documental. Elaboración propia. 


\begin{tabular}{|c|c|c|}
\hline \multicolumn{2}{|c|}{ Preguntas y variables } & \multirow{2}{*}{$\begin{array}{l}\text { Categorías } \\
\text { (Nombre) }\end{array}$} \\
\hline P1 & Nombre de la serie & \\
\hline P2 & Nombre del personaje & (Personaje) \\
\hline P3 & $\begin{array}{l}\text { Escalafón que ocupa el personaje } \\
\text { en el organigrama }\end{array}$ & Principal, Secundario, Aparición esporádica \\
\hline P4 & $\begin{array}{l}\text { Frecuencia de aparición del } \\
\text { personaje }\end{array}$ & (Ordinal, número capítulos) \\
\hline P5 & Edad del personaje & Bebé, Niña/o, Adolescente, Joven, Adulto, $3^{a}$ edad \\
\hline P6 & Estatura & Alto, Estatura media, Bajo, \\
\hline P7 & Peso & Con sobrepeso, Normal, Delgado \\
\hline P8 & Rasgos a destacar & (Codificación abierta) \\
\hline P9 & Clase social & (5 niveles) \\
\hline P10 & Nivel de estudios & (4 niveles) \\
\hline P11 & Ocupación & (Codificación abierta) \\
\hline P12 & Posición laboral & $\begin{array}{l}\text { Directiva/o, Subordinada/o de un hombre, Subordinada/o de } \\
\text { una mujer, Autónoma/o, Parada/o, Estudiante }\end{array}$ \\
\hline P13 & Descripción psíquica & $\begin{array}{l}\text { Inteligencia vs. Intuición, Autoestima alta vs. Baja, } \\
\text { Estabilidad emocional vs. Inestabilidad, Autonomía vs. } \\
\text { Dependencia, Sexualidad vs. Afectividad, Seriedad vs. } \\
\text { Frivolidad, Racionalidad vs. Tradicionalidad, Dominio vs. } \\
\text { Sumisión, Valentía vs. Miedo, Agresividad vs. Ternura, } \\
\text { Dinamismo vs. Pasividad, Objetividad vs. Subjetividad, } \\
\text { Frialdad vs. Sentimentalismo, Fuerza vs. Debilidad, Habilidad } \\
\text { vs. Torpeza, Profesionalidad vs. inexperiencia }\end{array}$ \\
\hline P14 & Marcas distintivas personaje & (Categorización abierta) \\
\hline P15 & $\begin{array}{l}\text { ¿Representa tareas o cargos } \\
\text { tradicionales de su sexo? }\end{array}$ & Sí, No \\
\hline P16 & $\begin{array}{l}\text { ¿Se puede asociar a algún } \\
\text { estereotipo fijado? }\end{array}$ & Sí, No \\
\hline P17 & ¿A cuál? & $\begin{array}{l}\text { Mujer como ama de casa clásica, Mujer como Ama de casa } \\
\text { moderna, Mujer como ama de casa o superwoman, Mujer } \\
\text { como madre de familia, Mujer como profesional clásica, } \\
\text { Mujer como triunfadora profesional, Mujer como profesional } \\
\text { actual, Mujer amazona, Mujer como acompañante u objeto } \\
\text { secundario, La jovencita, Mujer como cuerpo o símbolo } \\
\text { sexual, Mujer como símbolo de belleza, Mujer como objeto } \\
\text { decorativo, Mujer como objeto sexual. } \\
\text { Hombre infalible (triunfador), Hombre sensible, Hombre } \\
\text { perdedor, Hombre agresivo, Hombre valiente aventurero, } \\
\text { Hombre competitivo, Hombre objeto, Hombre como líder, } \\
\text { Hombre orgulloso y dominante, Hombre como padre } \\
\text { tradicional, Hombre como padre moderno, Hombre informal. }\end{array}$ \\
\hline
\end{tabular}

Tabla 2. Preguntas-categorías análisis narrativo. Elaboración propia 


\begin{tabular}{|c|c|c|}
\hline \multicolumn{2}{|c|}{ Preguntas y variables } & Categorías \\
\hline P1 & Nombre de la serie & (Nombre de la serie) \\
\hline P2 & No capítulo & (Codificación abierta. Variable de control) \\
\hline P3 & Cadena de emisión & (Nombre de la cadena) \\
\hline P4 & Fecha de emisión & (Codificación abierta. Variable de control) \\
\hline P5 & Lugar del emplazamiento & Casa/hogar, Trabajo/laboral, Ocio, Otros \\
\hline P6 & Entorno del emplazamiento & $\begin{array}{l}\text { Mayoritariamente mujeres, Mayoritariamente hombres, } \\
\text { Mixto, Mayoritariamente niñas/os, Ninguno }\end{array}$ \\
\hline P7 & Personaje del emplazamiento & $\begin{array}{l}\text { (Se muestra el listado de los personajes de cada serie en las } \\
\text { plantillas correspondientes a cada una de ellas) }\end{array}$ \\
\hline P8 & $\begin{array}{l}\text { ¿Representa una tarea } \\
\text { tradicional. atribuida a su sexo? }\end{array}$ & Sí, No \\
\hline P9 & $\begin{array}{l}\text { Rol que desempeña en el } \\
\text { emplazamiento }\end{array}$ & Usuario, Profesional, Entrevistador/narrador, Pasivo, Otro \\
\hline P10 & Tipo de emplazamiento & $\begin{array}{l}\text { Hiperactivo de valoración, Hiperactivo de mención, Verbal de } \\
\text { valoración, Verbal de mención, Activo, Pasivo principal, } \\
\text { Pasivo secundario }\end{array}$ \\
\hline P11 & $\begin{array}{l}\text { ¿Se da prescripción directa hacia } \\
\text { la compra? }\end{array}$ & Sí, No \\
\hline P12 & No de personajes que intervienen & (Número) \\
\hline P13 & Tipo de producto emplazado & $\begin{array}{l}\text { (Codificación abierta a priori. Tras la recogida de los datos } \\
\text { resulta la siguiente: Alcohol y tabaco, Bebidas no alcohólicas, } \\
\text { Alimentación, Limpieza y perfumería, Farmacia, Vehículos, } \\
\text { Textil y complementos, Finanzas, seguros y loterías, } \\
\text { Telefonía y aparatos de comunicación, Medios de } \\
\text { comunicación, Servicios, Deportes, Electrónica de consumo y } \\
\text { electrodomésticos, Otros) }\end{array}$ \\
\hline P14 & Marca emplazada & (Marca) \\
\hline
\end{tabular}

Tabla 3. Variables-categorías análisis de contenido. Elaboración propia 


\begin{tabular}{|l|l|l|}
\hline Etapa & Procedimiento aplicado & Resultados \\
\hline Pre-estudio & $\begin{array}{l}\text { Revisión de la literatura existente. } \\
\text { Selección universo adecuado. } \\
\text { Reunión de jueces (se analiza una parte del } \\
\text { material) }\end{array}$ & $\begin{array}{l}\text { Definiciones claras y código de los } \\
\text { instrumentos detallado. }\end{array}$ \\
\hline Estudio & $\begin{array}{l}\text { Formación de codificadores } \\
(2 \text { sesiones con 2 codificadores de forma } \\
\text { aislada) } \\
\text { Ensayo previo (test-test) } \\
(10 \%-20 \% \text { del material) }\end{array}$ & $\begin{array}{l}\text { a/pi superiores al 85\% en todas las } \\
\text { (Fiabilidad pertinente para aplicar la } \\
\text { herramienta) }\end{array}$ \\
\hline Post-estudio & $\begin{array}{l}\text { Test-reset } \\
(4 \text { meses después) }\end{array}$ & $\begin{array}{l}\text { a/pi superiores al 90\%. } \\
\text { (Herramientas fiables y estables) }\end{array}$ \\
\hline
\end{tabular}

Tabla 4. Procedimientos garantía de confiabilidad. Elaboración propia 


\begin{tabular}{|c|c|c|}
\hline Técnica & Estadístico & Resultados \\
\hline \multirow{4}{*}{$\begin{array}{l}\text { Test-test } \\
\text { Narrativo }\end{array}$} & $\%$ acuerdo & Superiores al $60 \%(>50 \%)$ \\
\hline & $a / p i$ & Superiores al $85 \%$ \\
\hline & P Spearman & Superiores a 0,70 . Asociación muy fuerte \\
\hline & V Kramer & Entre 0,50 y 0,70 . Asociaciones importantes \\
\hline \multirow{4}{*}{$\begin{array}{l}\text { Test-test } \\
\text { Contenido }\end{array}$} & $\%$ acuerdo & Superiores al $75 \%(>50 \%)$ \\
\hline & $a / p i$ & Superiores al $85 \%$ \\
\hline & P Spearman & Superiores a 0,86 . Asociaciones muy fuertes \\
\hline & V Kramer & Superiores a 0,70 . Asociaciones muy fuertes \\
\hline \multirow{4}{*}{$\begin{array}{l}\text { Test-reset } \\
\text { Narrativo }\end{array}$} & $\%$ acuerdo & Superiores al $80 \%(>50 \%)$ \\
\hline & $a / p i$ & Superiores al $90 \%$ \\
\hline & P Spearman & Superiores a 0,70 . Asociaciones muy fuertes \\
\hline & V Kramer & Entre 0,50 y 0,70 . Asociaciones importantes \\
\hline \multirow{4}{*}{$\begin{array}{l}\text { Test-reset } \\
\text { Contenido }\end{array}$} & $\%$ acuerdo & Superiores al $90 \%$ (> 50\%) \\
\hline & $a / p i$ & Superiores al $90 \%$ \\
\hline & P Spearman & Asociación perfecta $=1$ \\
\hline & V Kramer & Superiores a 0,90 . Asociaciones muy fuertes \\
\hline
\end{tabular}

Tabla 5. Resultados de estadísticos empleados. Elaboración propia

[Recibido: 5 de mayo de 2010. Aceptado (sin cambios): 26 de mayo de 2010.] 